\title{
Clinical Characteristics of Colorectal Carcinoid Tumors
}

\author{
Colonoscopy Study Group of Korean Society of Coloproctology \\ Colonoscopy Study Group of Korean Society of Coloproctology, Seoul, Korea
}

Purpose: The carcinoid tumor was recently categorized as a malignant disease due to its possibility of metastasis. This study was aimed to investigate the clinical characteristics and the metastatic rate of colorectal carcinoid tumors.

Methods: Charts were reviewed for 502 patients diagnosed with and treated for colorectal carcinoid tumors between January 2006 and December 2009. The location, size, depth and metastatic status of the tumors were collected.

Results: Including 24 synchronous tumors from 12 patients, 514 carcinoid tumors were removed. Most of them were found in the rectum (97.3\%). The male-to-female ratio was 1.38 to 1 , and mean age was $50.2 \pm 11.4$ years. The mean tumor size was $5.8 \pm 3.6 \mathrm{~mm}$. Less than 10 - $\mathrm{mm}$-sized tumors had a $1.95 \%$ lymph node metastatic rate; tumors with sizes from $10 \mathrm{~mm}$ to $20 \mathrm{~mm}$ and larger than $20 \mathrm{~mm}$ had $23.5 \%$ and $50 \%$ lymph node metastatic rates, respectively. Two cases had distant metastasis; one with a 22-mm-sized tumor metastasized to the liver, and the other with a 20-mm-sized tumor metastasized to the peritoneum. Among 414 patients who completed metastatic studies, $93.8 \%$ were classified as stage I, $0.9 \%$ as stage II, $4.8 \%$ as stage III and $0.5 \%$ as stage IV.

Conclusion: Colorectal carcinoid tumors smaller than $10 \mathrm{~mm}$ have a low rate of lymph node metastasis, but those sized 10 $\mathrm{mm}$ or larger incur significant risk. Further investigation regarding additional risk factors should be done to develop proper treatment guidelines for these tumors.

Keywords: Carcinoid tumor; Colorectal carcinoid tumor; Lymph node metastasis

\section{INTRODUCTION}

Carcinoid tumors are the most common neuroendocrine tumor in the gastrointestinal system. The incidence of carcinoid tumors varies depending on the organ. However, the rectum and the ileum are the most prevalent tumor sites in the gastrointestinal system [1].

Among gastrointestinal carcinoid tumors, colonic carcinoids have been reported to be relatively rare while rectal carcinoid tumors have been reported to be the most prevalent [1-4]. Generally, carcinoid tumors have been recognized as having low malignant potential. However, recent studies have shown consider-

Received: April 2, 2010 Accepted: August 25, 2010

Correspondence to: Jae-Bum Lee, M.D.

Department of Surgery, Daehang Hospital, 481-10 Bangbae-dong,

Seocho-gu, Seoul 137-820, Korea

Tel: +82-2-6388-8564, Fax: +82-2-6388-8673

E-mail: commonlee@naver.com

(C) 2011 The Korean Society of Coloproctology

This is an open-access article distributed under the terms of the Creative Commons Attribution NonCommercial License (http://creativecommons.org/licenses/by-nc/3.0) which permits unrestricted noncommercial use, distribution, and reproduction in any medium, provided the original work is properly cited. able rates of lymph node metastasis in carcinoid tumors. As well, the American Joint Council on Cancer (AJCC) has classified carcinoid tumors as malignant tumors [5].

The incidence of colorectal carcinoid tumors in Korea may be on the rise as the degree of screening colonoscopy increases [6, 7]. Although there have been reports of small-sized carcinoid tumors with lymph node metastasis, it is very difficult to assess national prevalence and rates of metastasis because previous domestic studies were limited to a single institute and had a limited volume of patients. Thus, the aim of this study is to investigate clinical characteristics focusing on the metastatic rate of colorectal carcinoid tumors through a large scale study.

\section{METHODS}

The colonoscopy study group of the Korean Society of Coloproctology conducted a retrospective study of colorectal carcinoid tumors in collaboration with eight institutes from January 2006 to December 2009. We gathered data such as age, gender, site, size, invasion depth and the presence of metastasis through the collection and review of medical records.

Rectal carcinoids were defined as tumors located within $15 \mathrm{~cm}$ 
of the anal verge while tumors more than $15 \mathrm{~cm}$ above the anal verge were regarded as colonic carcinoids. The tumors were classified into three groups according to tumor size: 1 ) a tumor diameter less than $10 \mathrm{~mm}, 2$ ) a $10-\mathrm{mm}$ to $20-\mathrm{mm}$ tumor diameter, and 3) a diameter larger than $20 \mathrm{~mm}$. Tumor sizes were confirmed through pathology reports. In the case of tumor sizes not being clearly described in the pathology reports, the size was determined according to the colonoscopy reports. Treatment methods were classified as endoscopic resection, transanal resection and radical operation.

In cases treated by radical operation, the presence of lymph node metastasis was determined by using a pathology report while in cases treated by local resection, including colonoscopic removal, metastatic status were assessed by radiologic studies (computer tomography [CT] scan). Cases which did not undergo metastatic work ups were excluded from the analysis of lymph node metastasis. If there were synchronous carcinoid tumors, only the largest tumors were included in the analysis of lymph node metastasis.

The tumor disease stages were determined according to the AJCC Cancer Staging 7th edition, revised in 2010. Statistical analysis was done with the Fisher's exact test and a P-value less than 0.05 was considered to be statistically significant.

Table 1. Clinical characteristics of colorectal carcinoid tumors

\begin{tabular}{lccc}
\hline & $\begin{array}{c}\text { Total } \\
(\mathrm{n}=514)\end{array}$ & $\begin{array}{c}\text { Rectum } \\
(\mathrm{n}=500)\end{array}$ & $\begin{array}{c}\text { Colon } \\
(\mathrm{n}=14)\end{array}$ \\
\hline Gender $^{\mathrm{a}}$ & & & \\
$\quad$ Male & $290(57.8)$ & $281(57.6)$ & $9(64.3)$ \\
$\quad$ Female & $212(42.2)$ & $207(42.4)$ & $5(35.7)$ \\
Age $^{\mathrm{a}}$ & $50.2 \pm 11.4$ & $50.0 \pm 11.3$ & $55.1 \pm 14.2$ \\
Size & $5.8 \pm 3.6$ & $5.7 \pm 3.5$ & $7.6 \pm 5.2$ \\
Treatment & & & \\
$\quad$ Endoscopic resection & $419(81.5)$ & $408(81.6)$ & $11(78.6)$ \\
$\quad$ Local resection & $54(10.5)$ & $54(10.8)$ & $0(0.0)$ \\
$\quad$ Radical operation & $41(8.0)$ & $38(7.6)$ & $3(21.4)$ \\
\hline
\end{tabular}

Values are presented as number (\%) or mean \pm SD.

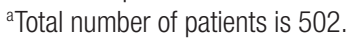

Table 2. Rate of lymph node metastasis in patients who underwent radical surgery

\begin{tabular}{lrrc}
\hline Tumor size $(\mathrm{mm})$ & $\begin{array}{c}\text { Total } \\
(\mathrm{n}=39)^{\mathrm{a}}\end{array}$ & $\begin{array}{c}\text { Rectum } \\
(\mathrm{n}=36)\end{array}$ & $\begin{array}{c}\text { Colon } \\
(\mathrm{n}=3)\end{array}$ \\
\hline$<10$ & $4 / 17(23.5)$ & $4 / 17(23.5)$ & - \\
$10-20$ & $12 / 19(63.2)$ & $11 / 16(68.8)$ & $1 / 3(33.3)$ \\
$>20$ & $2 / 3(66.7)$ & $2 / 3(66.7)$ & - \\
Total & $18 / 39(46.2)$ & $17 / 36(47.2)$ & $1 / 3(33.3)$ \\
\hline
\end{tabular}

The values in parentheses are presented as percent.

aTwo synchronous tumors in two patients (total number of carcinoid tumors is 41).

\section{RESULTS}

Five hundred two patients were treated for carcinoid tumors during the study period. In 12 patients, two carcinoid tumors were detected simultaneously (synchronous carcinoid tumor, 2.4\%); thus, the total number of resected carcinoid tumors was 514 . Fourteen carcinoid tumors were found in the colon, which accounts for $2.7 \%$, and 500 carcinoid tumors $(97.3 \%)$ were detected in the rectum. The ratio between males and females was 1.38:1, and the mean patient age was 50.2 years. The average tumor size was $5.8 \mathrm{~mm}$, and a regional resection, including colonoscopic treatments, was performed in 473 cases (92\%) (Table 1).

The result of pathological tests for 39 cases treated by radical surgery showed 18 cases of lymph node metastasis (46.2\%). The relationship of lymph node metastasis to tumor size for tumors smaller than $10 \mathrm{~mm}$ was $23.5 \%$, for tumors between $10 \mathrm{~mm}$ and $20 \mathrm{~mm}$, it was $63.2 \%$, and for tumors larger than $20 \mathrm{~mm}$, it was was $66.7 \%$ (Table 2).

Metastasis was assessed through CT scans or pathological examinations in 414 cases. When cases showing metastasis findings on the test were considered as positive for lymph node me-

Table 3. Rate of lymph node metastasis in 414 colorectal carcinoisa

\begin{tabular}{lccc}
\hline Tumor size $(\mathrm{mm})$ & $\begin{array}{c}\text { Total } \\
(\mathrm{n}=414)\end{array}$ & $\begin{array}{c}\text { Rectum } \\
(\mathrm{n}=402)\end{array}$ & $\begin{array}{c}\text { Colon } \\
(\mathrm{n}=12)\end{array}$ \\
\hline$<10$ & $7 / 359(1.95)$ & $7 / 352(1.99)$ & $0 / 7$ \\
$10-20$ & $12 / 51(23.5)$ & $11 / 46(23.9)$ & $1 / 5(20.0)$ \\
$>20$ & $2 / 4(50.0)$ & $2 / 4(50.0)$ & - \\
Total & $21 / 414(5.1)$ & $20 / 402(5.0)$ & $1 / 12(8.3)$ \\
\hline
\end{tabular}

The values in parentheses are presented as percent.

${ }^{a}$ Cases of radical operation or metastasis-evaluation using CT scan.

Table 4. Tumor-node-metastasis (TNM) staging of colorectal carcinoid tumors according to American Joint Council on Cancer, 7 th edition $(\mathrm{n}=414)$

\begin{tabular}{llc}
\hline \multicolumn{1}{l}{ TNM stage (clinical or pathologic) } & No. (\%) \\
\hline I & T1aN0M0 & $353(85.3)$ \\
Ila & T1bNOM0 & $35(8.5)$ \\
IIb & T2N0M0 & $4(0.9)$ \\
IIla & T3NOM0 & $0(0.0)$ \\
IIIb & T4NOM0 & $0(0.0)$ \\
IV & Any T, N1M0 & $20(4.8)$ \\
\hline
\end{tabular}

$\mathrm{T} 1$, tumor invades lamina propria or submucosa and size $\leq 2 \mathrm{~cm}$; T1a, tumor size $<1 \mathrm{~cm}$ in greatest dimension; T1b, tumor size 1 to $2 \mathrm{~cm}$ in greatest dimension; $\mathrm{T} 2$, tumor invades muscularis propria or size $>2 \mathrm{~cm}$ with invasion of lamina propria or submucosa; $\mathrm{T} 3$, tumor invades through the muscularis propria into the subserosa, or into non-peritonealized pericolic or perirectal tissues; T4, tumor invades peritoneum or other organs; N0, no regional lymph node metastasis; N1, regional lymph node metastasis; M0, no distant metastasis; M1, distant metastasis. 
tastasis, the aforementioned incidence according to tumor size for tumors smaller than $10 \mathrm{~mm}$ was $1.95 \%$ while for sizes between $10 \mathrm{~mm}$ and $20 \mathrm{~mm}$, it was $23.5 \%$, and for sizes larger than $20 \mathrm{~mm}$, it was $50.0 \%$. As tumor sizes increased, the metastasis incidence also increased (Table 3), and the frequency of lymph node metastasis in cases with carcinoid tumors smaller than 10 $\mathrm{mm}$ was confirmed to be significantly lower than in other groups $(\mathrm{P}<0.001)$. Regarding two cases with distant metastasis, one rectal case had metastasis to the liver, and the other colonic case involved peritoneal metastasis. The result of the TNM disease stage classification according to the AJCC is shown in Table 4.

\section{DISCUSSION}

Generally, the incidence of carcinoid tumors of the colon has been reported to be very low and comprises $4-8 \%$ of carcinoid tumors in the digestive system [1-4]. Colonic carcinoid tumors are detected in the cecum primarily, and two-thirds of colonic carcinoid tumors have been reported to occur in the right side of the colon [8]. More than half of carcinoid tumors of the colon are associated with lymph node metastasis or distant metastasis at the time of detection. Thus, prognosis is poor, with 5-year survival rates reported to be approximately $40-70 \%[1,9]$.

In our study, although it was limited to the colon and the rectum, the tumor incidence in the colon was $2.7 \%$, which is lower than the generally accepted incidence rate. As well, the number of cases treated with radical surgery was only three. Among the three, one case had lymph node metastasis, and another case had peritoneal metastasis. For the remaining eleven cases, the tumors were resected locally through colonoscopy. Using these data, estimating the lymph node metastasis rate in terms of colonic carcinoid tumors is very difficult, making risk predictions infeasible.

The proportion of colonoscopic resections for colonic carcinoid tumors in our series was relatively high in relation to general treatment guidelines, which recommend radical surgery for all colonic carcinoids. A possible explanation for this is that contrary to the usual experience of finding colonic carcinoid tumors at advanced stages, many colonic tumors in our series may were found incidentally at smaller sizes during diagnosis, deeming colonoscopic resection appropriate.

Most carcinoid tumors we detected were in the rectum, correlating with known findings as to the organ in which carcinoid tumors are detected most frequently. In our study, cases involving rectal carcinoid tumors totaled 500, accounting for approximately $97.3 \%$, with an average size of $5.7 \mathrm{~mm}$. The frequency of lymph node metastasis for rectal carcinoid tumors was found to be $5.0 \%$. Most rectal carcinoid tumors were treated by endoscopic or regional resection, such as transanal resection.

To evaluate the risk of metastasis, pathological examination of lymph nodes should be implemented. However, small rectal carcinoids are known to have little risk of metastasis, making local treatment desirable and reserving radical surgery for selected cases with risk factors associated with lymph node metastasis. Therefore, we substituted radiologic evaluation with CT scans for pathologic examination to confirm lymph node metastasis. Although CT scans do not reflect lymph node metastasis as accurately as a pathologic examination, if no sign of metastasis appears in the CT scan, we regard the case as negative for lymph node metastasis.

Four hundred four cases undergoing radical surgery and/or CT scan were included in the evaluation of metastasis. There was a direct correlation between tumor size and lymph node metastasis. The lymph node metastasis rate was approximately $2 \%$ if the tumor size was less than $10 \mathrm{~mm}$ and increased gradually up to $50 \%$ as the tumor size increased to larger than $20 \mathrm{~mm}$. For tumor sizes between $10 \mathrm{~mm}$ and $20 \mathrm{~mm}$, the risk of lymph node metastasis was as high as $24 \%$, which was similar to the results reported by Soga et al. [2]. This is thought to be a very important finding in deciding treatment modality. A consensus exists for which treatment method is appropriate for rectal carcinoid tumors with sizes between $10 \mathrm{~mm}$ and $20 \mathrm{~mm}$.

Carcinoid tumors develop in the mucosal gland and gradually infiltrate to the lamina propria, submucosal layer and deeper layer of the bowel wall. As the disease stage advances, carcinoid tumors may be accompanied by lymph node or distant metastasis. These clinical behaviors are the characteristics of malignant neoplasms. Thus, it is reasonable to regard colorectal carcinoids as malignant tumors. In diverse reports, lymph node metastasis and distant metastasis were reported and are thought to be tumors with a malignant potential comparable to that of an adenocarcinoma $[2,3]$. In the recently revised AJCC cancer staging, carcinoid tumors are classified as a malignant disease. The stage classifications are based on the size of the tumor, the involved layer of the bowel wall and the presence or absence of lymph node or distant metastasis. According to this staging system, our series of cases are classified into 388 stage I cases, 4 stage IIA cases, no stage IIB or IIIA cases, 20 stage IIIB cases, and 2 stage IV cases. The staging results reflect that most cases were early lesions and were detected incidentally by screening colonoscopy.

In cases of rectal carcinoids, the risks of lymph node metastasis and distant metastasis vary depending on the tumor size, making tumor size the most important factor in deciding on a treatment method. The reported risk of lymph node metastasis for rectal carcinoids smaller than $10 \mathrm{~mm}$ is usually less than $3 \%$; thus, regional treatment including colonoscopic resection is appropriate. However, radical surgery is recommended for tumors larger than $20 \mathrm{~mm}$, where the risk of metastasis is known to be $60-80 \%$. Treatment methods for tumors with sizes between 10 $\mathrm{mm}$ and $20 \mathrm{~mm}$ are controversial, and no established treatment guideline exists.

Known risk factors, other than tumor size, are tumor invasion beyond the proper muscle, the degree of cell differentiation and 
the mitotic rate. A population-based study in Japan reported $3.7 \%$ lymph node metastasis for rectal carcinoids with sizes of $5 \mathrm{~mm}$ and less and $10 \%$ for tumors with sizes less than $10 \mathrm{~mm}$ [2]. These results complicate deciding on treatment methods even in small-sized rectal carcinoids. Therefore, if lymph node metastasis is to be predicted accurately, additional studies on risk factors are required, and treatment methods should be determined considering various risk factors [9-11].

The large number of cases involved in this study aided in revealing meaningful data in terms of the risk of lymph node metastasis, yet, some limitations should be mentioned. First, it was a retrospective cross-sectional study and collected data were restricted to findings when tumors were detected and treated. Second, there may have been selection bias. In some institutions, only cases involving radical surgery were included. These cases may have had risk factors associated with lymph node metastasis. Third, we could not estimate the overall incidence, and this study lacked long-term follow-up results in terms of recurrence. This may play a role in underestimating the risk of lymph node metastasis. Additional studies on recurrence and the rate of metastasis through long-term follow-up are required in the future.

In rectal carcinoid tumors, the size of the tumor is directly related to the frequency of lymph node metastasis. In carcinoid tumors with sizes smaller than $10 \mathrm{~mm}$, the risk of lymph node metastasis was low; thus, it could be treated by regional treatments including colonoscopic resection. However, in carcinoid tumors with sizes larger than $10 \mathrm{~mm}$, the risk of lymph node metastasis increased remarkably. Especially, rectal carcinoid tumors with sizes between $10 \mathrm{~mm}$ and $20 \mathrm{~mm}$ showed a lymph node metastasis rate of $23.5 \%$, requiring treatment to be determined by considering various risk factors. Additional studies on the risk factors for lymph node metastasis are needed.

\section{CONFLICT OF INTEREST}

No potential conflict of interest relevant to this article was reported.

\section{ACKNOWLEDGEMENT}

We thank Dr. Hyun-Shig Kim, Dr. Hee-Cheol Jang (Seoul Song Do Hospital), Dr. Kyung-Su Han, Dr. Chang-Won Hong, Dr. Dae-Kyung Sohn (National Cancer Center), Prof. Won-Kyung Kang, Prof. Hye-Young Sung, Prof. Seong-Taek Oh (The Cath- olic University of Korea), Prof. Sung-Bum Kang, Prof. DuckWoo Kim (Seoul National University Bundang Hospital), Prof. Hyoung-Chul Park, Prof. Bong-Hwa Lee (Hallym University College of Medicine), Dr. Seung-Kyu Jeong, Dr. Dong-Hyun Choi, Dr. Yong-Taek Ko (Yang Hospital), Dr. Eun-Jung Lee, Dr. Eui-Gon Youk (Daehang Hospital), Dr. Seok-Won Lim (Hang Clinic of Coloproctology) for their contribution to this study. They provided valuable data for this study.

\section{REFERENCES}

1. Modlin IM, Lye KD, Kidd M. A 5-decade analysis of 13,715 carcinoid tumors. Cancer 2003;97:934-59.

2. Soga J. Early-stage carcinoids of the gastrointestinal tract: an analysis of 1914 reported cases. Cancer 2005;103:1587-95.

3. Konishi T, Watanabe T, Kishimoto J, Kotake K, Muto T, Nagawa $\mathrm{H}$, et al. Prognosis and risk factors of metastasis in colorectal carcinoids: results of a nationwide registry over 15 years. Gut 2007; 56:863-8.

4. Park YB, Kim JI, Ha BH, Cheung DY, Kim TH, Kim CH, et al. Endoscopic treatment of gastrointestinal carcinoid tumors. Korean J Med 2007;73:274-82.

5. Edge SB; American Joint Committee on Cancer. AJCC cancer staging manual. 7th ed. New York: Springer; 2010.

6. Park JB, Cha JM, Lee JI, Choe JW, Joo KR, Jung SW, et al. A case of small rectal carcinoid tumor with local lymph node metastases. Korean J Gastrointest Endosc 2009;38:52-6.

7. Chang JH, Kim SW, Chung WC, Kim YC, Jung CK, Paik CN, et al. Clinical review of gastrointestinal carcinoid tumor and analysis of the factors predicting metastasis. Korean J Gastroenterol 2007;50:19-25.

8. Raut CP, Kulke MH, Glickman JN, Swanson RS, Ashley SW. Carcinoid tumors. Curr Probl Surg 2006;43:383-450.

9. Maggard MA, O’Connell JB, Ko CY. Updated population-based review of carcinoid tumors. Ann Surg 2004;240:117-22.

10. Ramage JK, Goretzki PE, Manfredi R, Komminoth P, Ferone D, Hyrdel R, et al. Consensus guidelines for the management of patients with digestive neuroendocrine tumours: well-differentiated colon and rectum tumour/carcinoma. Neuroendocrinology 2008;87:31-9.

11. Kim BN, Sohn DK, Hong CW, Han KS, Chang HJ, Jung KH, et al. Atypical endoscopic features can be associated with metastasis in rectal carcinoid tumors. Surg Endosc 2008;22:1992-6. 